\title{
Structural defects in cubic semiconductors characterized by aberration-corrected scanning transmission electron microscopy
}

\author{
Yadira Arroyo Rojas Dasilva, Roksolana Kozak, Rolf Erni, Marta D. Rossell \\ Electron Microscopy Center, Empa, Swiss Federal Laboratories for Materials Science and \\ Technology, CH-8600 Dübendorf, Switzerland
}

\begin{abstract}
The development of new electro-optical devices and the realization of novel types of transistors require a profound understanding of the structural characteristics of new semiconductor heterostructures. This article provides a concise review about structural defects which occur in semiconductor heterostructures on the basis of micro-patterned Si substrates. In particular, one- and two-dimensional crystal defects are being discussed which are due to the plastic relaxation of epitaxial strain caused by the misfit of crystal lattices. Besides a few selected examples from literature, we treat in particular crystal defects occurring in $\mathrm{GaAs} / \mathrm{Si}, \mathrm{Ge} / \mathrm{Si}$ and $\beta$-SiC/Si structures which are studied by high-resolution annular dark-field scanning transmission electron microscopy. The relevance of this article is twofold; firstly, it should provide a collection of data which are of help for the identification and characterization of defects in cubic semiconductors by means of atomic-resolution imaging, and secondly, the experimental data shall provide a basis for advancing the understanding of device characteristics with the aid of theoretical modelling by considering the defective nature of strained semiconductor heterostructures.
\end{abstract}

Keywords: structural defects, twinning, dislocations, cubic semiconductors, zincblende structure, scanning transmission electron microscopy

\footnotetext{
* Corresponding author

Email address: marta.rossell@empa.ch (Marta D. Rossell)
}

Preprint submitted to Elsevier

September 23, 2016

(C) 2016. This manuscript version is made available under the Elsevier user license http://www.elsevier.com/open-access/userlicense/1.0/ 
2010 MSC: 00-01, 99-00

\section{Introduction}

The heterogeneous integration of high-quality compound semiconductor materials with silicon technologies holds enormous potential in the field of optoelectronics. It should enable unprecedented levels of performance in a wide 5 range of optoelectronic devices such as high-electron-mobility transistors, highefficiency solar cells, and monolithically integrated lasers. The interest in integrating alternative semiconductor materials with $\mathrm{Si}$ resides in the possibility to take advantage of both the exceptional physical properties of the former and the well-established low-cost Si complementary metal oxide semiconductor (CMOS) technology. However, despite advancements in the growth technology, the fabrication of high-quality structures still remains a challenge $[1,2,3,4,5]$. Defects in the overgrown semiconductor structures originate from the different structural and thermal characteristics of the two dissimilar materials, as well as from non-perfect planar substrates. This leads to the formation of a broad spectrum of structural defects during growth. Typical crystal defects originating from the interface comprise threading and misfit dislocations, stacking faults, twin boundaries and antiphase boundaries. Obviously, a high density of these defects results in severe degradation of the optoelectronic properties, thereby limiting the performance of the devices. For example, dislocations are generally assumed to cause carrier recombination $[6,7]$, while stacking faults and antiphase boundaries are known to cause unacceptable leakage currents in power devices and/or to slow down their operating speed due to decreased charge carrier mobility at the boundaries [8].

A crucial step towards the optimization of the optoelectronic device structures is the investigation of the defect formation mechanisms. Hence, the detailed atomic study of the local structure at the interface between the semiconductor epilayers and the Si substrates is of particular interest. For this, transmission electron microscopy (TEM) is ideally suited as it provides the spa- 
tial resolution to study the structure of materials on the atomic level. Over the past 30 years, extended defects in semiconductor materials have been intensively studied by conventional and high-voltage high-resolution TEM [1, 9, 10, 11, 12]. However, it was experimentally challenging to resolve the exact atomic-scale structure due to the lack in spatial resolution and/or image delocalization in conventional phase contrast micrographs. Thus, "holographic" reconstruction techniques were indispensable to increase the spatial resolution $[13,14,15]$. Alternatively, image simulations based on structural models were performed to validate the image interpretation $[11,12]$.

More recently, the implementation of spherical-aberration correctors in scanning transmission electron microscopes (STEM) $[16,17,18]$ has lead to dramatic 40 improvements in lateral resolution. These instruments are now capable of routinely producing images in the deep sub-Ångström range [19]. Thus, the dumbbell structure in crystalline Si along $<110>$ and $<112>$ samples can be clearly resolved, which is particularly useful for the characterization of defects [20]. Additionally, by using a high-angle annular dark-field (HAADF) detector

45 it is possible to produce images which show contrast that approximately scales with the square of the atomic number, hence the name Z-contrast imaging. This allows to discriminate, for example, between the lighter $\mathrm{Cd}$ atomic columns from the heavier Te columns in the CdTe compound semiconductor. Recent studies of low-dimensional semiconductor structures and devices by aberration-corrected 50 STEM clearly demonstrate the power of this technique for investigating defects and interfaces $[3,21,22,23,24,25,26,27,28,29]$. It has proven to be decisive both in the detection of novel types of defects but also in the advancement of our understanding of seemingly basic crystal-structure defects.

The scope of this review is to summarize and organize dispersed information about this topic. In this review, which is intended for the non-specialist, we provide an overview of one- and two-dimensional lattice defects typically found in face-centered cubic semiconductors. Zero-dimensional (or point) defects and three-dimensional defects, such as clusters, inclusions, voids or second-phase particles, are not treated here. It shall serve to readers who are concerned with 
defect analysis of atomically resolved images of face-centered cubic semiconductors, and it shall provide a phenomenological overview of common and less frequent one- and two-dimensional defects which can impact the performance of novel semiconductor devices.

\section{Experimental details}

Unless otherwise stated, all experimental images were taken using a double spherical aberration-corrected JEOL JEM-ARM200F microscope equipped with a cold field-emission electron source operating at $200 \mathrm{kV}$. In STEM mode, a convergence semiangle of 25 mrad was used in combination with an annular dark field (ADF) detector with inner and outer collection semiangles of 90 and 370 mrad, respectively. A Gaussian low-pass filter for noise reduction was applied to all images.

Samples for the HAADF-STEM analysis were prepared by means of either a FEI Helios NanoLab 600i or FEI Helios NanoLab 450S focussed ion beam (FIB) operated at accelerating voltages of 30 and $5 \mathrm{kV}$. Additionally, Si/Ge specimens

75 [30] were prepared by mechanical polishing and dimple grinding, followed by ion-milling with $\mathrm{Ar}^{+}$ions using a Fischione Model 1050 TEM-Mill operating at low voltages and grazing incidence to achieve electron transparency.

\section{Configuration of planar defects}

The most commonly observed planar (2D) defects in cubic semiconductors by using high-resolution TEM are twin boundaries (TBs) and stacking faults (SFs) [31]. They are caused by discontinuities in the ...AaBbCcAaBbCc... stacking sequence of $\{111\}$-type close-packed layers in diamond or, its ordered variant, zincblende structure. Additionally, compound semiconductor layers grown on elemental semiconductor substrates, like $\mathrm{Si}$ or Ge, are also susceptible to form

85 antiphase boundary (APB) defects. In the following sections, the characteristics of these defects are discussed. 
- Twin boundaries. Twin boundaries are formed along the intersection of two differently oriented lattices/parts of the crystal. They are called coherent (CTB) if there is a perfect (one-to-one) matching of lattice planes across the bound90 ary as it is shown in Fig. 1a,b. They form a $\sim 35^{\circ}$ angle with the $\{110\}$-type planes, indicating that it is a $\{111\}$-CTB. Thus, an angle of $\sim 70^{\circ}$ is obtained between the $\{110\}$-type planes at each side of the CTB. In heteroatomic cubic semiconductors depending on the polarity of the atoms, CTBs can be divided into mirror (also called reflection or para-twin) and rotational (ortho-twin) [32]. When two crystal parts share a common mirror plane -which also corresponds to a $\sim 70^{\circ}$ lattice rotation about the common [110] axis- a mirror CTB is created, whereas when they are related to each other in addition by a $180^{\circ}$ rotation about [110] (or by a $\sim 60^{\circ}$ twist around [111]) a rotational CTB is formed. HAADFSTEM images of rotational and mirror CTBs in $\beta$-SiC with overlaid schematic models are shown in Fig. 1a,b, respectively. Mirror twins possess a mirror symmetrical arrangement of $\mathrm{Si}-\mathrm{C}$ dumbbells along the common axis (with stacking sequence ...AaBbC $\| \mathrm{CbBaA} . .$.$) , whereas rotational twins are characterized by an$ asymmetric polarity (...AaBbC $\| \mathrm{cBbAa} \ldots)$, where $\|$ indicates the twin boundary position. For zincblende semiconductors, mirror twinning is far less dominant due to the much higher cost in energy to form the necessary homopolar bonding.

A twin boundary is called incoherent (ITB) if there is no perfect matching of lattice planes across it. An example of ITB is shown in Fig. 1c. It is constructed of periodically arranged units each of which is built of 7-, 5- and a pair of 6-fold rings. The ITB forms a $\sim 13^{\circ}$ angle with the $\{110\}$ planes, indicating that it is of $\Sigma 19\{331\}$-type [33]. Thus, an angle of $\sim 26^{\circ}$ is obtained between the $\{110\}$-type planes at each side of the ITB.

An interesting structural modification on the pure zincblende structure that is often found in nanowires is periodic twinning, also referred to as polytypism $[34,35,36,37,38]$. Polytypes are a special case of polymorphs, where the closed-packed crystal structures differ in one dimension only. Thus, the geometry of the bilayers is maintained but the layer stacking sequence of the overall crystal structure is varied. In this context, the zincblende structure can be 

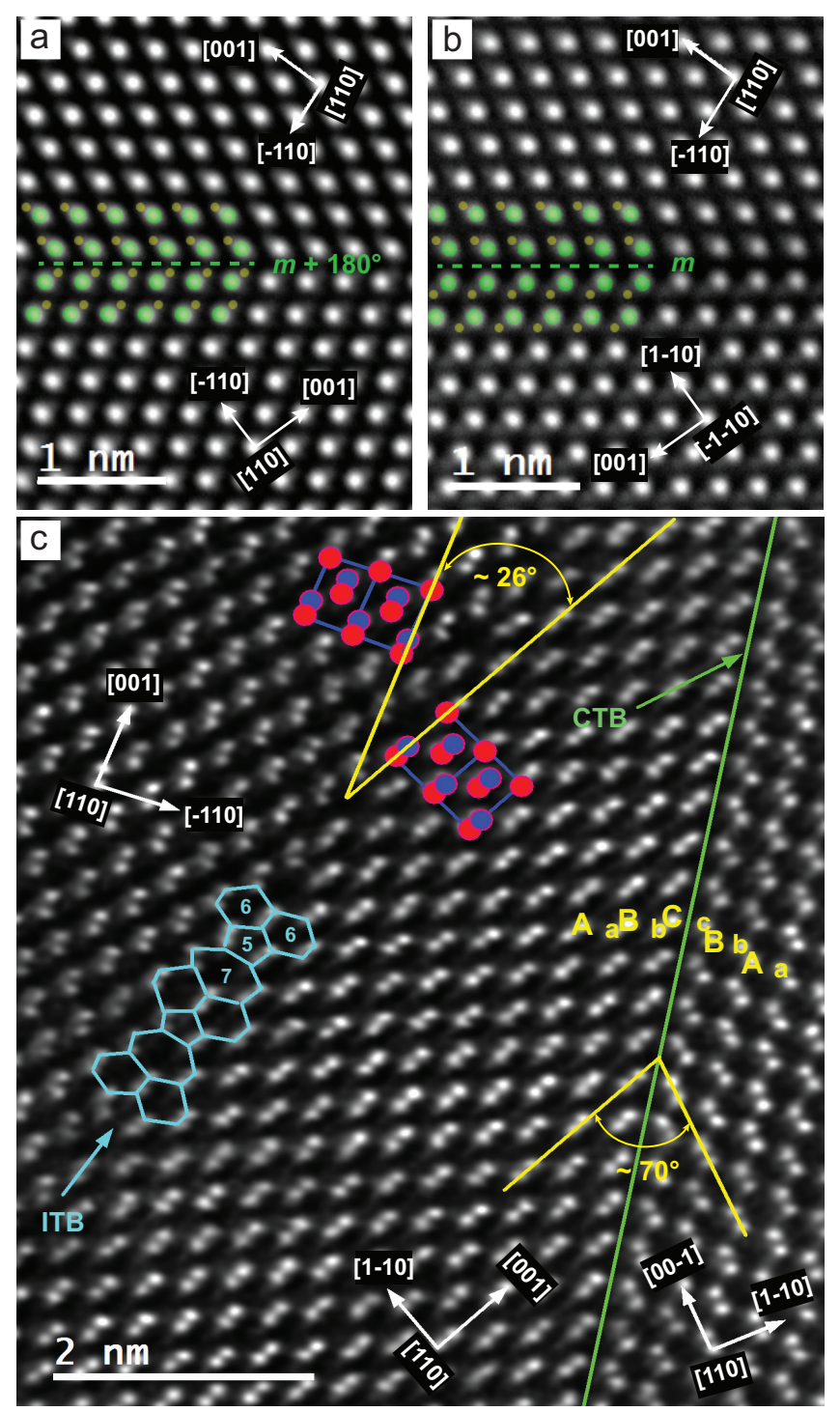

Figure 1: Twin boundaries in zincblende semiconductor crystals. Coherent twin boundaries in $\beta$-SiC with overlaid atomic models (with the $\mathrm{Si}$ and $\mathrm{C}$ columns in green and yellow) representing the two types of CTBs: a) rotational, and b) mirror twins. c) Coherent and incoherent twin boundaries in GaAs marked as CTB and ITB, respectively. The angles between the $\{110\}$-type planes at each side of the CTB and ITB are indicated in yellow. The GaAs unit cell is displayed as ball-and-stick models with the Ga and As columns shown in red and blue, respectively. 
considered a polytype with a repeating ...AaBbCcAaBbCc... sequence, that is the 3C polytype in the Ramsdell notation [39], where $\mathrm{C}$ is used to denote cubic structures. Wurtzite, with a repeating ...AaBbAaBb... sequence for the $2 \mathrm{H}$ variant ( $\mathrm{H}$ stands for hexagonal), is the polytype with the shortest period. The other polytypes represent hexagonal $(\mathrm{H})$ or rhombohedral $(\mathrm{R})$ combinations of these stacking sequences having an $n$ number of bilayers in the primitive cell [40]. Important examples are the $4 \mathrm{H}$ and $6 \mathrm{H}$ polytypes with four and six double layers, respectively $[41,42,23,43]$. Silicon carbide $(\mathrm{SiC})$ is one of the few compounds, which form such stable and long-range modifications [44]. The recurrent insertion of twin defects in the cubic zincblende structure results in multi-quantum-well structures dramatically influencing the electronic and optoelectronic properties of nanowires $[38,45,46]$.

- Stacking faults. A stacking fault is formed by the dissociation of a perfect dislocation into partial dislocations in order to reduce its strain energy at the expense of forming a planar defect. The simplest examples of stacking faults observed in cubic semiconductors are presented in Fig. 2. An intrinsic stacking fault (ISF) is formed when one $\{111\}$-type plane is removed from the perfect crystal. Then the stacking sequence is ...AaBbCcAaBbCc... An extrinsic stacking fault (ESF) is created when one additional close-packed plane is inserted and results in the stacking sequence ...AaBbCc $\underline{B^{*} b^{*}} \mathrm{AaBbCc} \ldots$ They easily form during non-optimized growth in zincblende semiconductors due to their low formation energy. Additionally, it is well known that the stacking fault energy decreases with increasing ionicity of the bonding [47, 48].

- Antiphase boundaries. Antiphase boundaries (APBs) represent a special case of $2 \mathrm{D}$ defects in zincblende semiconductors, where the polarity of the crystal is inverted on either side of the boundary. Thus, the two domains differ by a $90^{\circ}$ rotation about the [001] axis or inversion operations. They are characterized 145 by the formation of anti-site bonds, i.e. cation-cation and anion-anion bonds. In bulk, APBs might be produced by the climb of a $1 / 2[111]$ edge dislocation with line direction [110] [49]. However, they are most commonly observed in 

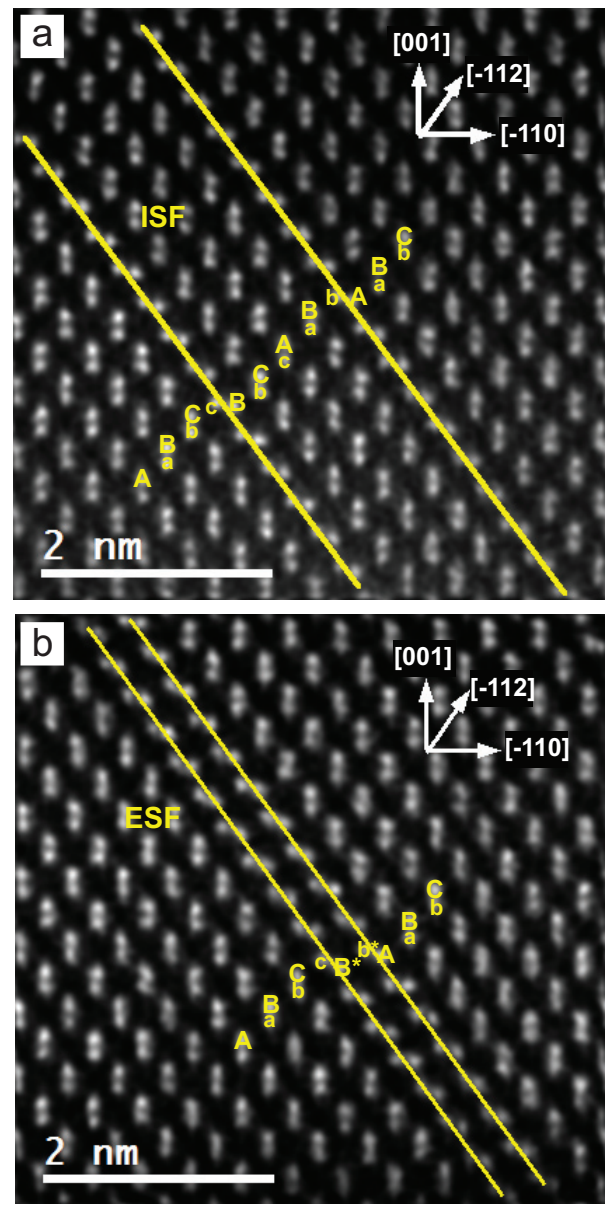

Figure 2: Stacking faults in GaAs. a) Two intrinsic and b) one extrinsic stacking faults with their corresponding stacking sequences. 
overgrown layers because of the presence of the unavoidable presence of singleatomic-height steps at the (001) surface of group-IV semiconductors. APBs can either propagate through the entire film along $\{110\}$-type planes or kink to higher-index planes and self-annihilate [50] as shown in Fig. 3a. The former case is the most energetically favorable, while the $\{111\}$ APB has the highest energy [51]. Both $\{112\}$ and $\{113\}$ APBs have an intermediate value of formation energy that makes these planes good candidates for the APB kinking and annihilation [51, 52]. Figure 3b shows a HAADF-STEM image of an APB in a GaSb film initiated from the interface with $\mathrm{Si}$ and propagated to the epilayer surface [3]. The red dashed line highlights the "wrong" bonds between same-type atoms.

\section{Configuration of dislocations}

Dislocations are line defects; they locally disturb the regular ordering of the atoms along their line in the solid. Thus, the interatomic bonds are significantly distorted in the immediate vicinity of the dislocation line, or so-called "dislocation core", while the distortion decays rapidly with distance from the core. Dislocations participate in the growth of crystals and also in the interface structure between two crystals. Indeed, semiconductor materials are often grown in heteroepitaxy, that is the substrate material and the material grown on top are different and usually there is a certain lattice mismatch, i.e. a difference between the lattice constants of the two materials. This lattice mismatch is accommodated by the formation of misfit dislocations (MDs) at the interface, located either at the substrate or at the grown layer.

Figure 4 illustrates the presence of dislocations both inside a Ge crystal and at a $\mathrm{Ge} / \mathrm{Si}$ interface, together with their corresponding $\varepsilon_{x y}$ strain field maps obtained by geometrical phase analysis (GPA) [53]. In particular, Fig. 4a,b show two parallel dislocations lying on perpendicular slip planes in close proximity within a Ge crystal and the strain field interaction between them: both dislocation cores exhibit a compression region (in blue) and a tensile region (in 

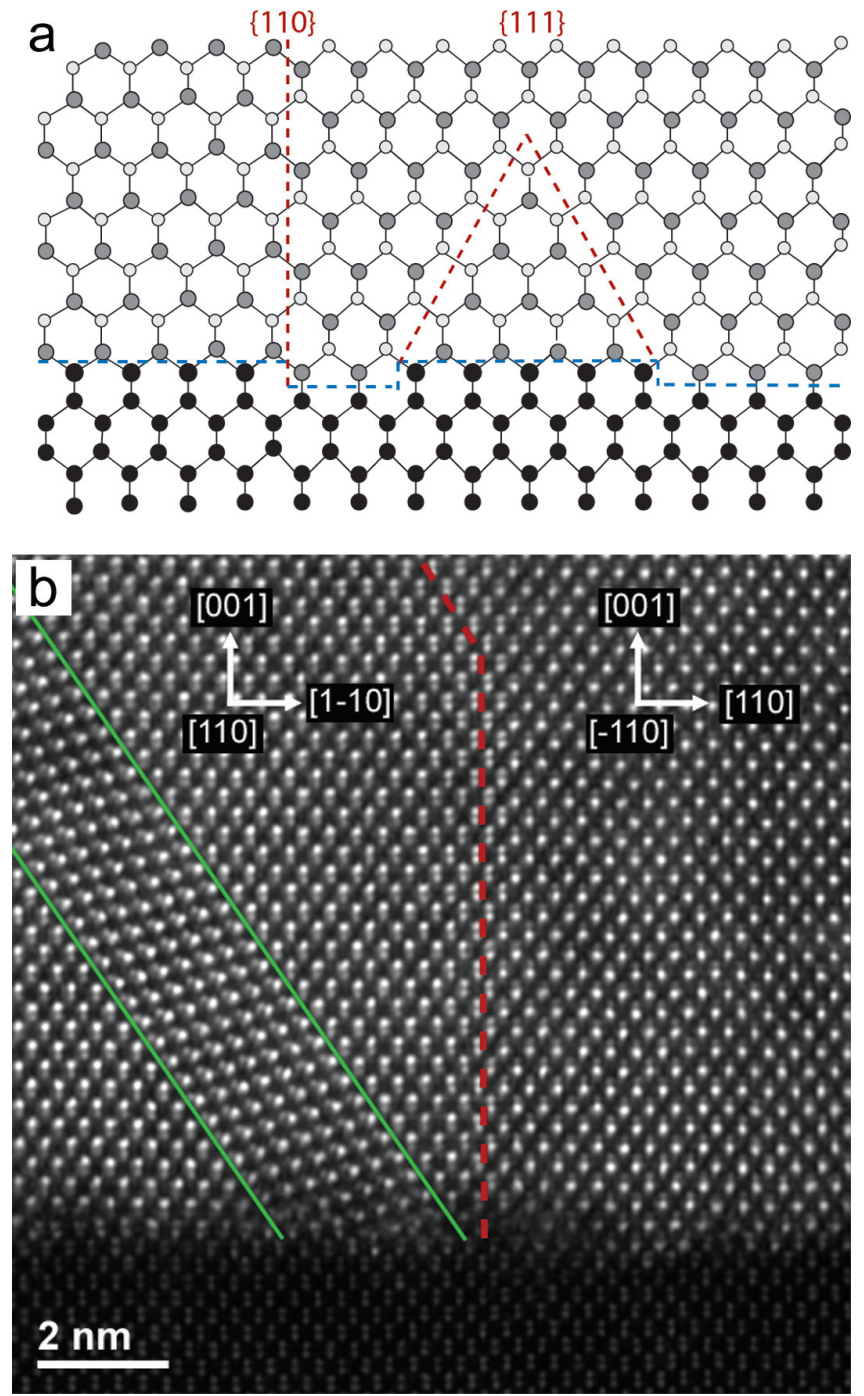

Figure 3: Antiphase boundaries in zincblende semiconductor films. a) Model of APB formation in the $\{110\}$ and $\{111\}$ lattice planes of a zincblende film grown on a group-IV semiconductor (001) substrate due to the presence of monolayer steps on the substrate surface. b) HAADF-STEM image of an APB in a GaSb film grown onto a not perfectly planar $\mathrm{Si}$ substrate. The two antiphase domains differ by a $90^{\circ}$ rotation about the axis perpendicular to the substrate/film interface. A red dashed line highlights the position of the APB. The position of two rotational twin boundaries (CTBs) is indicated by green continuous lines. The figure from panel $\mathrm{b}$ is adapted from [3]. 

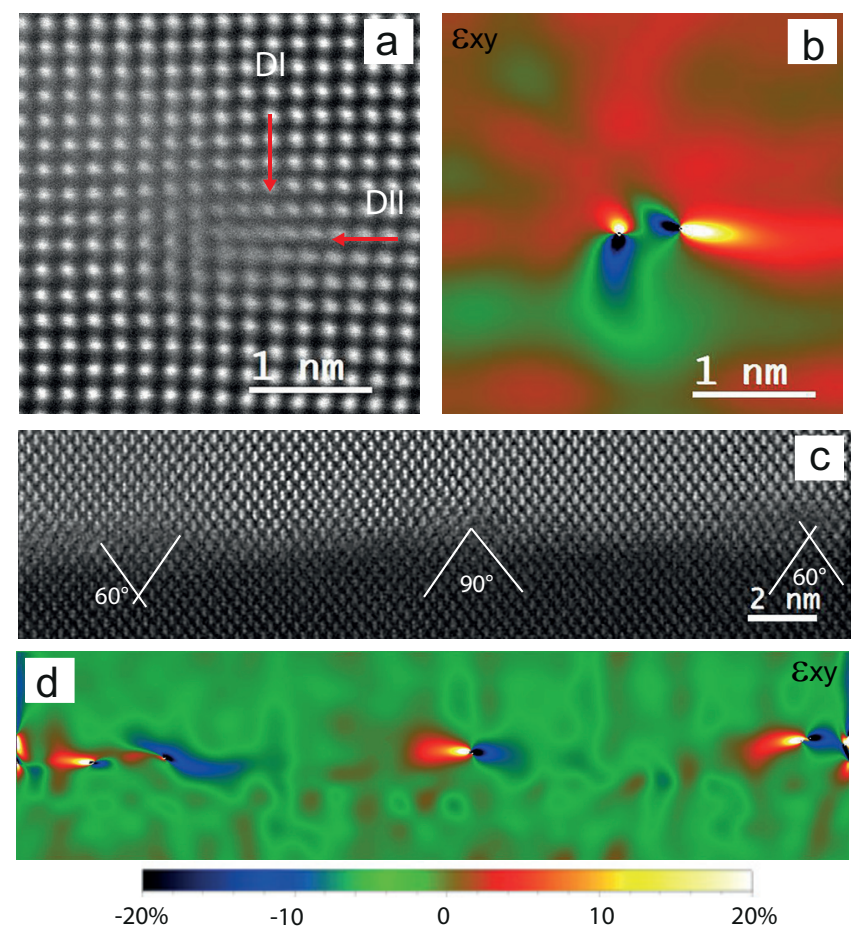

Figure 4: Dislocations participating in the growth of crystals and in the interface structure between two crystals. a,b) Two perpendicular dislocations (marked with red arrows) in close proximity within a Ge crystal and corresponding $\varepsilon_{x y}$ strain field image. c,d) Misfit dislocations (marked by white lines) at the Ge/Si interface and corresponding $\varepsilon_{x y}$ strain field image. 
yellow) [30]. Similar butterfly-like shapes are observed at the $\mathrm{Si} / \mathrm{Ge}$ interface (Fig. 4c,d). They are MDs (marked with white arrows) resulting from the $4.2 \%$ lattice mismatch between $\mathrm{Ge}$ and $\mathrm{Si}$, and are identified as pairs of perfect glissile $60^{\circ}$ and perfect sessile $90^{\circ}$ MDs (a more detailed description of these type of dislocations is given in the following sections). The number of atomic planes between the MDs is not constant. It varies between 20 to 40 planes depending on the area of observation. Theoretically, for the total relaxation of the misfit strain one MD should be introduced every 24 planes $\left[n=a_{S i} /\left(a_{G e}-a_{S i}\right), n=\right.$ number of planes, $a=$ lattice constant, $a_{G e}=0.5658 \mathrm{~nm}$ and $a_{S i}=0.5431 \mathrm{~nm}$ ]. Experimentally, the number of $\{111\}$ planes between the MDs is slightly larger with an average of $26 \pm 0.5$ planes between dislocations. Therefore, not enough MDs are incorporated to fully relax the strain plastically at the selected growth temperatures [54].

A dislocation is characterized by two parameters: the direction of the dislocation line, denoted by $\xi$, and its Burgers vector $\vec{b}$. The Burgers vector $\vec{b}$ describes the magnitude and direction of the lattice distortion, and $\xi$ is the line in the slip plane that separates the regions where slip has occurred from those where it has not. In Fig. 5, the dislocation line $\xi$ runs along the bottom of the extra half-plane of atoms. The Burgers vector $\vec{b}$ is determined by drawing the Burgers circuit, that is by drawing an atom-to-atom circuit enclosing the dislocation (Fig. 5). Here, we employ the FS/RH convention [49]. First, a starting point $\mathrm{S}$ is chosen arbitrarily. Afterwards, a clockwise circuit is built around the dislocation line passing through $1,2,3$, and ending at point $\mathrm{F}$, with the same amount of unit cells in the vertical and horizontal directions. If $\mathrm{F}$ does not coincide with $\mathrm{S}$, at least one dislocation is present in the area of the circuit. The Burgers vector $\vec{b}$ (green arrow) is obtained by closing the circuit F-S.

Depending on the angle between $\xi$ and $\vec{b}$, dislocations are classified as edge, screw and mixed. An edge dislocation is formed when an extra half-plane of 205 atoms is inserted in the crystal structure; then $\xi$ is perpendicular to $\vec{b}\left(90^{\circ}\right)$. A screw dislocation is described as a single helicoid surface where the atoms are arranged in a helical pattern normal to the direction of the stress; $\xi$ is parallel to 


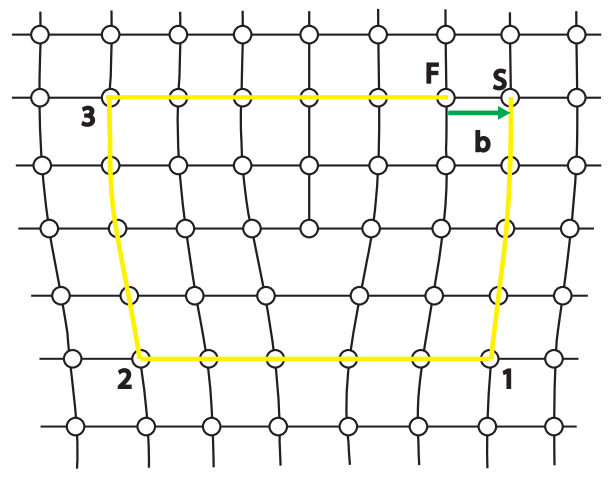

Figure 5: Burgers circuit around an edge dislocation.

$\vec{b}\left(0^{\circ}\right)$. A mixed dislocation has an arbitrary angle between $\xi$ and $\vec{b}$, consisting of both screw and edge character.

Besides, depending on the magnitude of the Burgers vector $\vec{b}$ dislocations are also classified as perfect dislocations and partial (PDs) dislocations. Thus, if $\vec{b}$ is equal to a unit lattice vector the dislocation is referred as to a perfect dislocation, whereas if $\vec{b}$ is less than a unit lattice vector the dislocation is called a partial dislocation, and is usually associated with one more dislocation.

The energy of a dislocation is proportional to the square of its Burgers vector $b^{2}$. By calculating the energy of dislocations it is possible to determine if it is energetically favorable for two dislocations to react and combine in order to form a new one, such as $b_{1}{ }^{2}+b_{2}{ }^{2}>b_{3}{ }^{2}$ (Frank rule) [55]. Here, the energy of the new dislocation $\left(b_{3}{ }^{2}\right)$ is smaller than the two dislocations reacting $\left(b_{1}{ }^{2}+b_{2}{ }^{2}\right)$.

\subsection{Perfect dislocations}

In the face-centered cubic system, the Burgers vector of a perfect dislocation can either be $\vec{b}=1 / 2<110>$ or $\vec{b}=<001>$, both of which are lattice vectors between two neighboring lattice atom sites. However, dislocations with $\vec{b}=1 / 2<110>$ have half the energy of those with $\vec{b}=<001>$, and are therefore more favorable. Depending on the line direction $\xi$, perfect dislocations are classified into $60^{\circ}$ (mixed), edge $\left(90^{\circ}\right)$ and screw $\left(0^{\circ}\right)$ dislocations. 


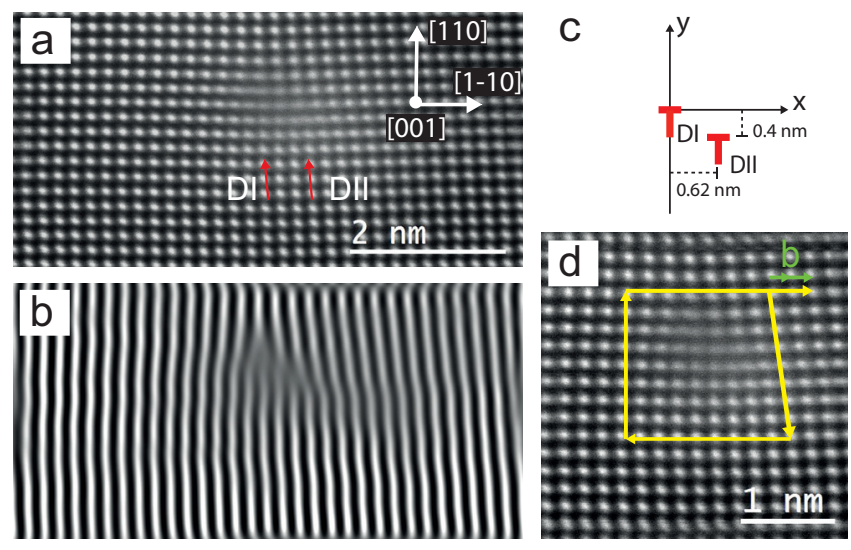

Figure 6: Pair of edge threading dislocations. a) Parallel edge dislocations (marked as DI and DII). b) Bragg-filtered image obtained using the (220) reflection. c) Schematic representation of the position of the edge dislocations. d) Burgers circuit around the dislocations and resulting Burgers vectors indicated with green arrows.

- Edge dislocations. Edge threading dislocations (TDs) are observed in the $\mathrm{Ge} / \mathrm{Si}$ system; they are created by the change in direction of $60^{\circ}$ threading dislocations [30]. They are observed in the Ge layer as two parallel edge TDs with parallel or perpendicular Burgers vectors. Figure 6a shows a HAADFSTEM image along the [001] direction of two parallel edge TDs (indicated as DI and DII) with parallel Burgers vector. The Bragg-filtered image in Fig. 6b is extracted from Fig. 6a using the $(2 \overline{2} 0)$ reflection; it shows the two dislocations as two half-planes located at $(2 \overline{2} 0)$ planes separated by $0.62 \mathrm{~nm}$. They lie on parallel (220) slip planes with a separation of $0.4 \mathrm{~nm}$ (Fig. 6c). The Burgers circuit around the TDs is shown in Fig. 6d using the FS/RH convention. The Burgers vector closing the circuit is $\vec{b}=1 / 2[1 \overline{1} 0]$ for each dislocation.

- Screw dislocations. The motion of a screw dislocation is the result of shear stress, but the dislocation line movement is perpendicular to the applied stress and the atom displacements, rather than parallel. Because its screw characteristic, a distinction is made between left-handed and right-handed screw dislocations. Figure 7 a shows a drawing of a glide creating a right-handed screw dislocation, where the glide plane is displayed in gray and the Burgers vector $\vec{b}$ 
(green arrow) is directed in the direction of the sense vector $\xi$. For left-handed projection of the atomic positions (Fig. $7 \mathrm{~b}$ ) along the dislocation line shows a zigzag pattern, a characteristic feature of screw dislocations. This zigzag pattern is due to the shear displacement from the upper part of the crystal along the slip plane with respect the lower part. The larger dark grey atoms indicate while smaller black and light gray circles mark the position of the upper and lower part of the crystal, respectively. Thus, the atoms at the left side of Fig. $7 \mathrm{~b}$ have not moved from their original position while the atoms at the right side have moved to a new position in the lattice and have re-established bonds. The atoms in the middle are shifted from their positions in the perfect crystal.

At low magnification, screw threading dislocations (STDs) have been observed in Ge layers grown on $\mathrm{Si}(001)$ using dark-field two-beam condition [57], being about 2-3 times more abundant than edge threading dislocations. They are characterized by a Burgers vector $\vec{b}=[001]$ and a dislocation line lying along the [001] direction. In annealed Ge, the STDs dissociate into two $60^{\circ}$ dislocations with $\vec{b}=1 / 2<110>$ gliding in the $\{111\}$ planes and disappear [58]. At high resolution, screw dislocations in a f.c.c. material have only been observed in Pt nanoparticles [59] with a clearly visible zigzag pattern. Atomically resolved screw dislocations in hexagonal close-packed (h.c.p.) GaN have also been demonstrated by HAADF-STEM [60, 61]. However, to the best of our knowledge, screw dislocations in f.c.c. semiconductors have not been imaged by HAADF-STEM so far.

- $60^{\circ}$ dislocations. In the face-centered cubic system, mixed dislocations are called $60^{\circ}$ dislocations because the angle between the Burgers vector $\vec{b}$ and the line direction $\xi$ is $60^{\circ}$. Figure 8 shows a HAADF-STEM image of the Ge/Si interface with two $60^{\circ}$ misfit dislocations (white lines): they are in different $\{111\}$ planes and their line $\xi$ is along [110]. The Burgers circuits around the dislocations are depicted by yellow and orange arrows, and their Burgers vectors are 

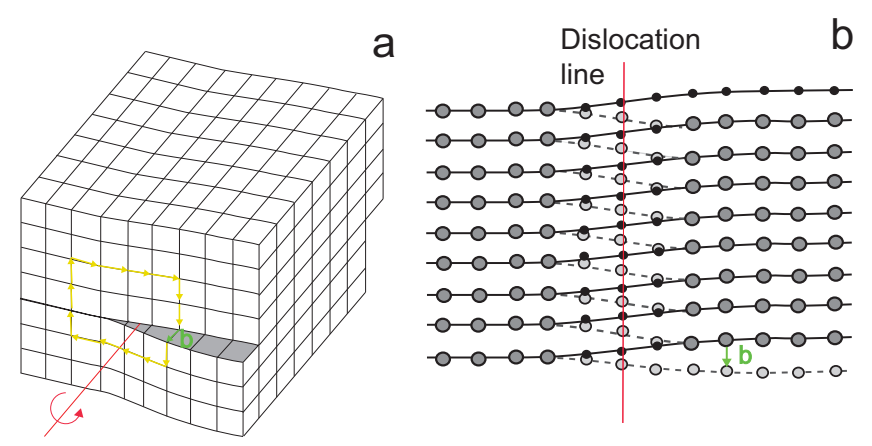

Dislocation line

Figure 7: Model of a right-handed screw dislocation. a) Motion of the screw dislocation resulting from shear stress. b) Projection of the atomic positions along the dislocation line with the characteristic zigzag pattern.

indicated by green arrows. They are $\vec{b}=1 / 2[10 \overline{1}]$ and $\vec{b}=1 / 2[01 \overline{1}]$, respectively.

275 STEM observations on $60^{\circ}$ misfit dislocations have been previously reported at GaAs/Si [62] and GaSb/GaAs interfaces [63, 64].

\subsection{Partial dislocations}

Partial dislocations are extended dislocations associated with stacking faults. The two typical types of partial dislocations (PDs) in the face-centered cubic materials are Shockley (SPDs) and Frank partial dislocations (FPDs). SPDs are formed by splitting of $60^{\circ}$ or screw perfect dislocations, whereas FPDs are associated to interstitial or vacancy $\{111\}$ planes. Thus, SPDs are glissile (they lie in the $\{111\}$ planes) and FPDs are sessile (they lie in different planes than $\{111\})$. The Burgers vectors for the SPDs and FPDs are $\vec{b}=1 / 6<112>$ and ${ }_{285} \vec{b}=1 / 3<111>$, respectively.

- Shockley partial dislocations. Figure 9a shows two Shockley partial dislocations in Ge. The general Burgers circuit is depicted by orange arrows giving a null vector. A null vector indicates that the perfect dislocation which splits does not have an edge component. The half-planes and the single layers are pointed by red arrows (1 and 2) and red circles, respectively. The Burgers circuits around each of the two partials are drawn by yellow arrows. For a partial 


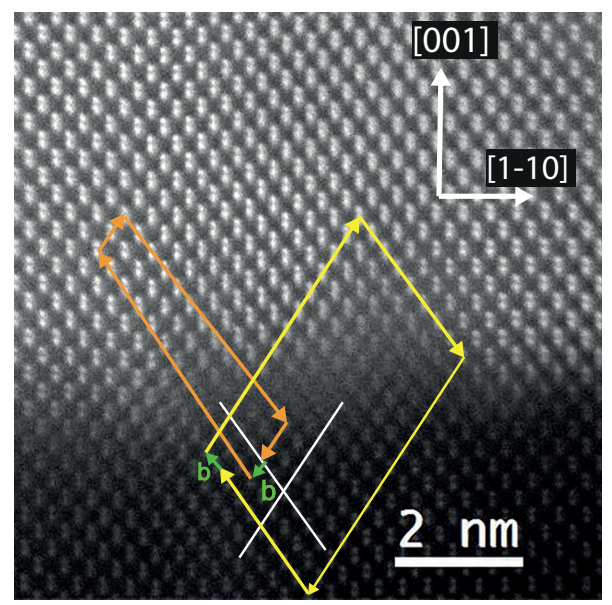

Figure 8: $60^{\circ}$ misfit dislocations in $\mathrm{Ge} / \mathrm{Si}$. Yellow and orange arrows are used to indicate the Burgers circuits around the dislocations, while the Burgers vectors are shown as green arrows.

dislocation, the Burgers circuit must begin and end on the plane of the stacking fault, and go around the dislocation core [49]. The Burgers circuit does not end on an atomic position, since for partial dislocations $\vec{b}$ is less than a unit lattice vector. The Burgers vectors are shown with green arrows; their magnitude corresponds to $1 / 3$ of the distance between two closest atom sites along the $<112>$ direction [65]. This value corresponds to a $30^{\circ}$ Shockley partial dislocation. The cores of the dislocations also correspond to $30^{\circ}$ dislocations having a half-plane ending with a single column [66]; one of the dislocation cores is enlarged in Fig. 9c. The Burgers vectors are $\vec{b}_{1}=1 / 6[211]$ and $\vec{b}_{2}=1 / 6[12 \overline{1}]$ for PD1 and PD2, respectively. Most likely, the splitting dislocation is a screw dislocation because the two $30^{\circ}$ PDs associated to an intrinsic stacking fault have a null general Burgers circuit. The possible splitting is given by reaction (1) having an energy reduction $\left(\frac{a^{2}}{2}>\frac{a^{2}}{3}\right)$, therefore the splitting is favorable:

$$
\frac{1}{2}[110] \rightarrow \frac{1}{6}[211]+\frac{1}{6}[12 \overline{1}]
$$

Figure 9e shows a model [66] of the dissociation of a $60^{\circ}$ perfect dislocation into a $30^{\circ}$ and a $90^{\circ}$ partial dislocations with an intrinsic stacking fault (blue dashed line). The core of the $30^{\circ} \mathrm{PD}$ of the Fig. $9 \mathrm{c}$ is in an agreement with 

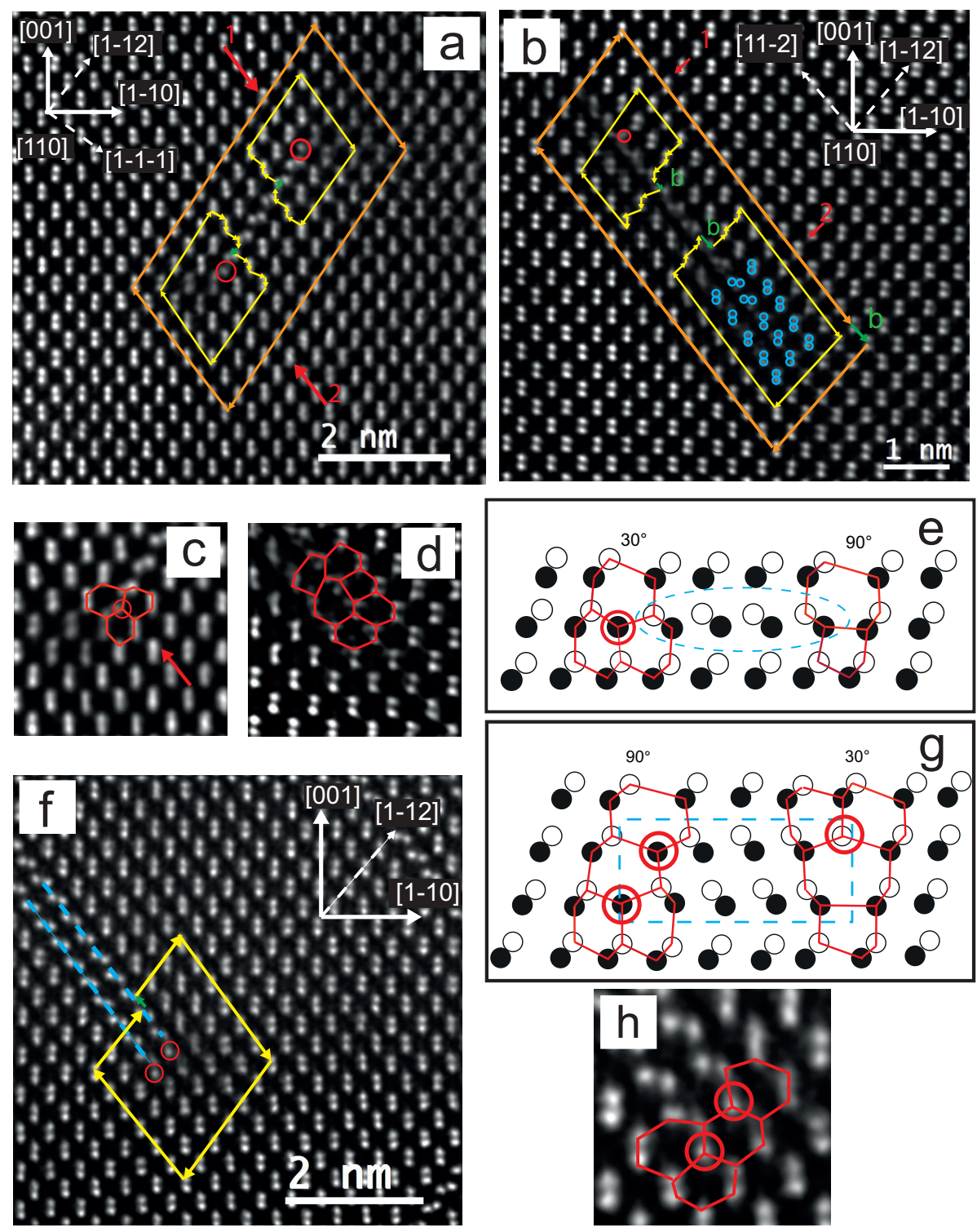

Figure 9: Shockley partial dislocations. a) Two $30^{\circ}$ partial dislocations with an intrinsic stacking fault. b) A $30^{\circ}$ and a $90^{\circ}$ partial dislocations with an intrinsic stacking fault. cd) Cores of the $30^{\circ}$ and $90^{\circ}$ PDs from panels a and b, respectively. e) Model of an intrinsic stacking fault associated with a $30^{\circ}$ and a $90^{\circ}$ partial dislocations. f) A $90^{\circ}$ partial dislocation with an extrinsic stacking fault. g) Model of an extrinsic stacking fault associated with a $90^{\circ}$ and a $30^{\circ}$ partial dislocations. h) Core of the $90^{\circ} \mathrm{PD}$ from panel $\mathrm{f}$. 
the model of Fig. 9e for a $30^{\circ} \mathrm{PD}$, which shows half-plane ending with a single column. Figure 9b corresponds to an intrinsic stacking fault bounded by two partial dislocations. The Burgers circuits of the partials are depicted by yellow arrows and the Burgers vectors are indicated by green arrows. The magnitude of the Burgers vector for partial 1 and 2 corresponds to $1 / 3$ and $2 / 3$ of the distance between two atoms along the $<112>$ direction, respectively. Therefore, dislocation 1 corresponds to a $30^{\circ}$ partial and dislocation 2 is a $90^{\circ}$ partial, having Burgers vectors $\vec{b}=1 / 6[21 \overline{1}]$ and $\vec{b}=1 / 6[1 \overline{1} \overline{2}]$, respectively. The blue circles outline the arrangement of the atoms close to the core of the $90^{\circ} \mathrm{PD}$. An enlarged image of the core of the $90^{\circ} \mathrm{PD}$ is shown in Fig $9 \mathrm{~d}$, and is in agreement with the $90^{\circ} \mathrm{PD}$ model in Fig. 9e consisting of two adjacent 5 and 7 -membered atom rings. The general Burgers circuit is depicted by orange arrows around the two partial dislocations giving a general $\vec{b}=1 / 2[101]$, which corresponds to a $60^{\circ}$ perfect dislocation. The splitting reaction of the $60^{\circ}$ dislocation is given by equation (2); it shows that the splitting is favorable as a reduction of energy takes place $\left(\frac{a^{2}}{2}>\frac{a^{2}}{3}\right)$ :

$$
\frac{1}{2}[10 \overline{1}] \rightarrow \frac{1}{6}[21 \overline{1}]+\frac{1}{6}[1 \overline{1} \overline{2}]
$$

Figure 9f shows the structure of an extrinsic stacking fault (blue lines) bounded by a partial dislocation. The Burgers circuit (yellow arrows) around the partial dislocation results in a Burgers vector whose magnitude is $2 / 3$ of the distance between two lattice sites along the $<112>$ direction. Therefore, it corresponds to a $90^{\circ}$ Shockley partial dislocation. When associated to an extrinsic stacking fault, $90^{\circ}$ PDs show two unpaired columns in the dislocation core (marked with red circles). Thus, the $90^{\circ} \mathrm{PD}$ can be considered to be equivalent to two $30^{\circ}$ PDs with intrinsic stacking faults lying on adjacent glide planes $[49,67]$. The core structure of the $90^{\circ} \mathrm{PD}$ is shown in Fig. 9h and consists of 6-membered atom rings. This configuration is in agreement with the model proposed by $\mathrm{Lu}$ and Smith [66] for a $90^{\circ} \mathrm{PD}$ bounded by an extrinsic 335 stacking fault (Fig. 9g). 

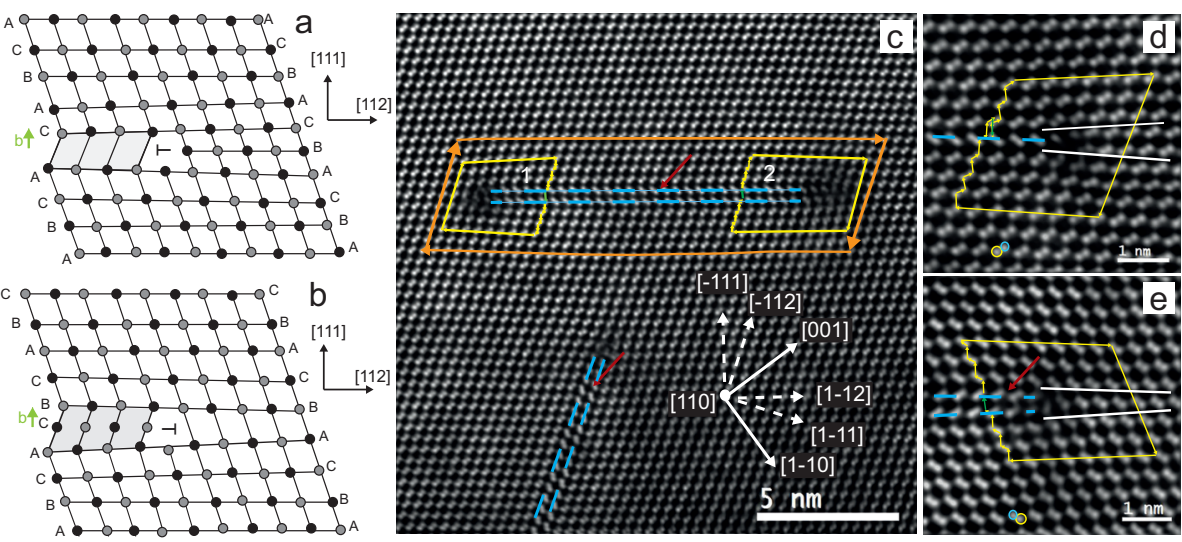

Figure 10: Frank partial dislocations. a) Model of a negative Frank partial dislocation. b) Model of a positive Frank partial dislocation. c) Positive sessile Frank partial dislocations in CdTe. Blue dashed lines indicate the position of the stacking faults. The Burgers circuits and vectors around each partial dislocation are depicted by yellow and green arrows, respectively. The closed Burgers circuit (orange arrows) indicate that the projected component of the Burgers vector is zero along the [110] viewing direction. d) Negative sessile Frank partial dislocation in CdTe. An extra vacancy plane is condensing on an intrinsic stacking fault. e) Positive sessile Frank partial dislocation in CdTe. The red arrow shows the position of the extra plane. The small blue and big yellow circles in panels $\mathrm{d}$ and e indicate $\mathrm{Cd}$ and $\mathrm{Te}$ columns, respectively. Figures from panels c-e are adapted from [22].

- Frank partial dislocations. Frank partial dislocations are produced by the condensation of lattice vacancies or interstitial atoms in the form of discs [68]. A negative Frank partial dislocation (Fig. 10a) is associated with an intrinsic stacking fault ...ABCACABC... (removal of a layer), whereas a positive Frank partial dislocation (Fig. 10b) is associated with an extrinsic stacking fault ...ABCACBCABC... (insertion of a layer). The Burgers vector of a Frank partial dislocation is normal to the $\{111\}$ plane of the fault, and the magnitude of the Burgers vector is equal to the change in spacing produced by one close-packed layer [55]. They are edge sessile partial dislocations with $\vec{b}=1 / 3<111>$.

345 Figure 10c shows two extrinsic stacking faults (blue dashed lines) bounded by partial dislocations in CdTe [22]. The stacking faults are lying on the (111) and (11̄1) planes; the extra plane in each fault is indicated by a red arrow. 
The Burgers circuits around each partial dislocation are depicted by yellow arrows. The Burgers vectors (green arrows) are $\vec{b}=1 / 3[1 \overline{1} \overline{1}]$ and $\vec{b}=1 / 3[\overline{1} 11]$ for dislocation 1 and 2, respectively. The general Burgers vector is depicted by orange arrows giving null vector, meaning that the dislocation does not have an edge component. The splitting dislocation is a screw dislocation or a dipole dislocation (two partials with opposite Burgers vectors).

HAADF-STEM images of a negative and a positive sessile Frank partial in Fig. 10d show that there is an extra vacancy plane condensing in a intrinsic stacking fault, whereas in Fig. 10e there is an interstitial atom plane giving rise to an extrinsic stacking fault [22]. The Burgers circuits are depicted by yellow arrows resulting in $\vec{b}=1 / 3[1 \overline{1} \overline{1}]$ and $\vec{b}=1 / 3[\overline{1} 11]$ for the negative and positive 360 Frank partial dislocations, respectively. These partial dislocations agree with the models shown in Figs. 10a and b.

\subsection{Interaction between dislocations}

- Lomer dislocations. Lomer or Lomer lock dislocations (90 $\mathrm{MDs})$ are formed when two perfect dislocations moving in different $\{111\}$ planes meet forming an acute angle $\left(70^{\circ}\right)$ and interact. They are sessile edge dislocations lying on $\{001\}$ planes.

In the $\mathrm{Ge} / \mathrm{Si}$ system, Lomer dislocations are observed at the interface due to the mismatch between the Ge film and the Si substrate or near the interface as shown in Fig. 11a. The red arrows point to two $60^{\circ}$ MDs that meet in 370 the $\{001\}$ plane; they are interacting and forming a Lomer dislocation. The FS/RH Burgers circuit is depicted by the yellow arrows and the Burgers vector by the green arrow. According to the circuit, the Burgers vector of the Lomer dislocation is $\vec{b}=1 / 2[\overline{1} 10]$. The Lomer dislocation has its line along the [110] direction and lies on the (001) plane. Therefore, it is considered to be an edge 

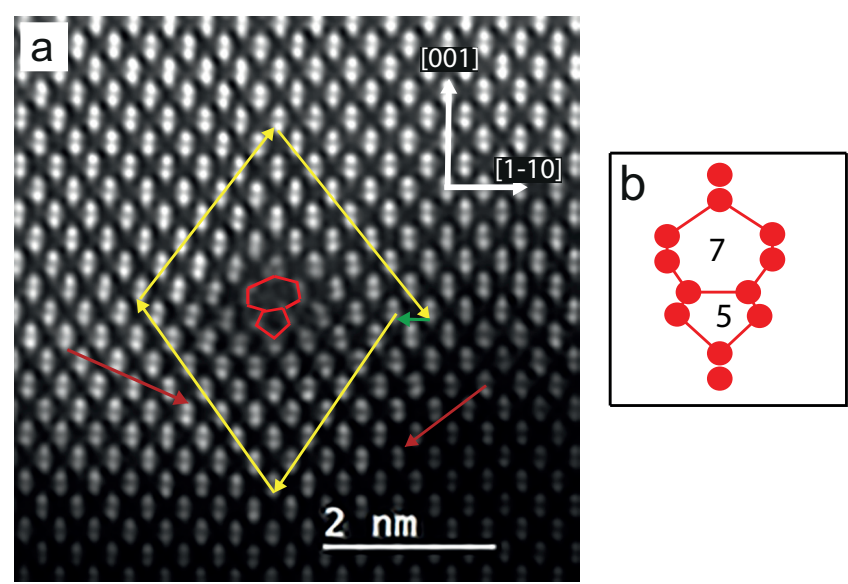

Figure 11: Lomer dislocation in the Ge/Si system. a) Burgers circuit (yellow arrows) and resulting Burgers vector (green arrow). b) Model of the core of the Lomer dislocation. Red arrows point out the half-planes.

dislocations is:

$$
\frac{1}{2}[\overline{1} 0 \overline{1}]+\frac{1}{2}[011] \rightarrow \frac{1}{2}[\overline{1} 10]
$$

The Burgers vectors of the two $60^{\circ}$ dislocations are given at the left side of the equation, while the resulting Burgers vector of the Lomer dislocation is found at the right side. This reaction is favorable as the energy is reduced by

$50 \%\left(a^{2}>\frac{a^{2}}{2}\right)$. The core of the Lomer dislocation is also shown in the model of Fig. 11b; it consists of one 5 and one 7 -membered atom rings [63, 64, 21], and agrees well with the core of the Lomer dislocation in Fig. 11a.

- Stair rod dislocations. Stair rod dislocations, also called Lomer-Cottrell locks, are partial dislocations which are formed when a partial dislocation bends from one glide plane to another or interacts with a partial dislocation in another slip plane [49]. The identification of the Burgers vector of a stair rod dislocation is obtained by subtracting the Burgers vectors of the two circuits around the faults [69]. Thus, one circuit is built around one stacking fault (SF1) starting and finishing in the fault. The other circuit is drawn around both the SF1 and 

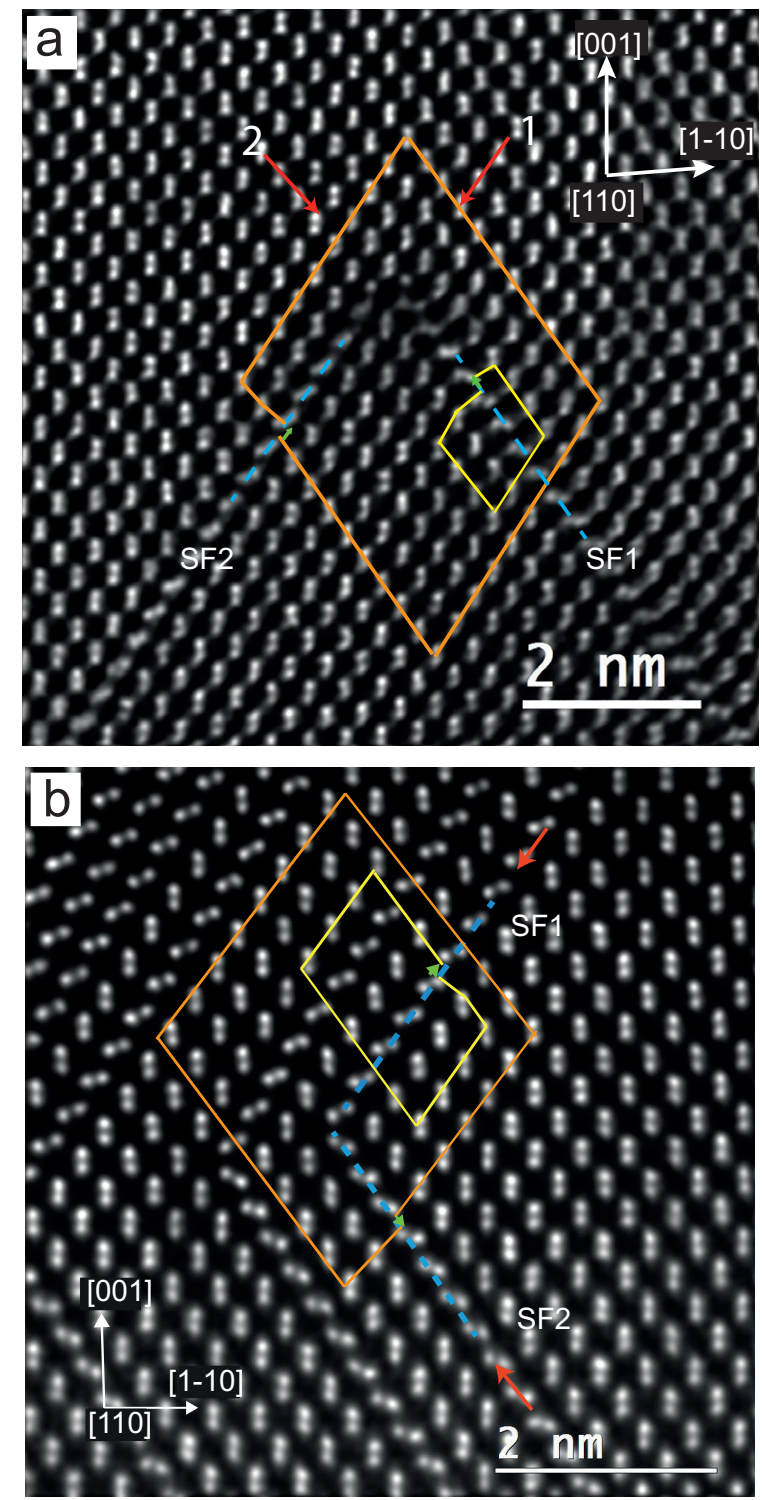

Figure 12: Stair rod dislocations. a) Stair rod dislocation in Ge located about $18 \mathrm{~nm}$ above the $\mathrm{Ge} / \mathrm{Si}$ interface having $\vec{b}=1 / 6[1 \overline{1} 0]$. b) Stair rod dislocation in GaAs located about $5.5 \mathrm{~nm}$ above the GaAs/Si interface having $\vec{b}=1 / 3[001]$. Yellow and orange arrows are the Burgers circuits around the faults. Red arrows point out the half-planes. 

considering that the crystal is unfaulted at the position of SF1.

In the $\mathrm{Ge} / \mathrm{Si}$ system, stair rod dislocations are often found at the intersection line of two stacking faults lying on different glide planes with an acute angle of $70.5^{\circ}$ (Fig. 12a). The stacking faults (blue dashed lines) annihilate each other The Burgers vectors obtained by the circuits are $\vec{b}=1 / 6[211]$ (yellow circuit) and $\vec{b}=1 / 6[\overline{1} \overline{2} \overline{1}]$ (orange circuit), which correspond to $30^{\circ}$ partial dislocations. The Burgers vector of the stair rod dislocation is $\vec{b}=1 / 6[1 \overline{10}]$ and lies on the (001) plane.

In GaAs crystals grown on $\mathrm{Si}$, stair rod dislocations are also frequently observed (Fig. 12b). They connect two stacking faults (blue dashed lines) with an obtuse angle. The red arrows point out the half-planes introduced by the stacking faults. The Burgers vectors obtained by drawing the circuits are $\vec{b}=1 / 6[211]$ and $\vec{b}=1 / 6[\overline{2} \overline{1} 1]$ for the yellow and orange circuit, respectively. Thus, the BurgThis dislocation is also called Hirth lock dislocation.

\section{Conclusions}

The increased resolution, contrast and signal-to-noise ratio of aberrationcorrected electron microscopy have made possible the direct investigation of lattice defects in semiconductors with unprecedented clarity. Recent reports on novel types of defects, as well as on revisited defects, demonstrate the power of aberration-corrected HAADF-STEM for the atomic-scale characterization of defects and to confirm structural models of, e.g., dislocation cores which were previously conjectured based on theory.

This review organizes the dispersed information about this topic and provides an overview about structural defects which occur in face-centered cubic semiconductors. In the first part, planar (2D) defects encompassing twin boundaries, stacking faults and antiphase boundaries are described. In the second part, 
line (1D) defects are discussed. In particular, perfect and partial dislocations, and their interactions are thoroughly described.

The detailed structural analysis of the atomic positions at lattice defects is of increasing importance in determining their impact on device performance. Thus, based on the experimental data, models of the defects can be constructed and ab-initio methods, like density functional theory, can be used to calculate their electronic properties $[27,70]$. Knowledge about the structure of lattice defects and their electronic activity are of paramount importance to assess the behavior of novel semiconductor devices, to improve synthesis and growth processes and to optimize device performance.

\section{Acknowledgements}

The authors are thankful to many colleagues for the samples used in this study, including Hans von Känel, Fabio Isa, Ivan Prieto, Thomas Kreiliger, Giovanni Isella, Oliver Skibitzki, Capellini Giovanni, and Thomas Schröder. We gratefully thank Shahrzad Hosseini Vajargah and Gianluiggi Botton for providing the image reproduced in Fig. 3; and Chen Li and Andy R. Lupini for providing the image reproduced in Fig. 10. This research was supported by the Swiss National Science Foundation under the project number 200021L_153558 and the Sinergia project NOVIPIX. Access to the TEM facilities at IBM ResearchZurich, Switzerland, under the IBM/Empa Master Joint Development Agreement is gratefully acknowledged.

\section{References}

[1] V. Narayanan, S. Mahajan, K. Bachmann, V. Woods, N. Dietz, Stacking faults and twins in gallium phosphide layers grown on silicon, Phil. Mag. A 82 (2002) 685-698. doi:10.1080/01418610208243196.

[2] A. Beyer, J. Ohlmann, S. Liebich, H. Heim, G. Witte, W. Stolz, K. Volz, Gap heteroepitaxy on si(001): Correlation of si-surface structure, gap 
growth conditions, and si-iii/v interface structure, J. Appl. Phys. 111 (2012) 083534. doi:10.1063/1.4706573.

[3] S. Hosseini Vajargah, S. Woo, S. Ghanad-Tavakoli, R. Kleiman, J. Preston, G. Botton, Atomic-resolution study of polarity reversal in gasb grown on si by scanning transmission electron microscopy, J. Appl. Phys. 112 (2012) 093101. doi:10.1063/1.4759160.

[4] J. Ma, X. Zhu, K. Wong, X. Zou, K. Lau, Improved gan-based led grown on silicon (111) substrates using stress/dislocationengineered interlayers, J. Cryst. Growth 370 (2013) 265-268. doi:10.1016/j.jcrysgro.2012.10.028.

[5] M. Borg, H. Schmid, K. Moselund, G. Signorello, L. Gignac, J. Bruley, C. Breslin, P. D. Kanungo, P. Werner, H. Riel, Vertical iii-v nanowire device integration on si(100), Nano Lett. 14 (2014) 1914-1920. doi: $10.1021 / \mathrm{n} 1404743 \mathrm{j}$.

[6] J. Seto, The electrical properties of polycrystalline silicon films, J. Appl. Phys. 46 (1975) 5247-5254. doi:10.1063/1.321593.

[7] A. Sutton, R. Balluffi, Interfaces in crystalline materials, Oxford Science Publications, New York.

[8] R. Alcotte, M. Martin, J. Moeyaert, R. Cipro, S. David, F. Bassani, F. Ducroquet, Y. Bogumilowicz, E. Sanchez, Z. Ye, X. Y. Bao, J. B. Pin, T. Baron, Epitaxial growth of antiphase boundary free gaas layer on 300 mm si(001) substrate by metalorganic chemical vapour deposition with high mobility, APL Mater. 4 (2010) 046101. doi:10.1063/1.4945586.

[9] D. Biegelsen, F. Ponce, A. Smith, J. Tramontana, Initial stages of epitaxial groth of gaas on (100) silicon, J. Appl. Phys. 61 (1986) 1856-1859. doi:10.1063/1.338029. 
[10] F. Ernst, P. Pirouz, Formation of planar defects in the epitaxial growth of gap on si substrate by metal organic chemical-vapor deposition, J. Appl. Phys. 64 (1988) 4526-4530. doi:10.1063/1.341280.

[11] P. Lu, D. Smith, Direct imaging of cdte(001) surface reconstructions by high-resolution electron microscopy, Surf. Sci. 254 (1991) 119-124. doi : 10.1016/0039-6028(91) 90644-8.

[12] J. Stirman, P. Crozier, D. Smith, F. Phillipp, G. Brill, S. Sivananthan, Atomic-scale imaging of asymmetric lomer dislocation cores at the ge/si(001) heterointerface, J. Appl. Phys. 84 (2004) 2530. doi:10.1063/1.1697625.

[13] P. Schiske, Zur frage der bildrekonstruktion durch fokusreihen, Proceedings of the fourth European Regional Conference on Electron Microscopy, Rome 1 (1968) 145-146.

[14] E. Kirkland, B. Siegel, N. Uyeda, Y.Fujiyoshi, Digital reconstruction of bright field phase contrast images from high resolution electron micrographs, Ultramicroscopy 5 (1980) 479-503. doi:10.1016/0304-3991(80)90048-0.

[15] W. Coene, G.Janssen, M. Op de Beeck, D. Van Dyck, Phase retrieval through focus variation for ultra-resolution in field-emission transmission electron microscopy, Phys. Rev. Lett. 69 (1992) 3743-3746. doi:10.1103/PhysRevLett.69.3743.

[16] O. Krivanek, N. Dellby, A. Spence, R. Camps, L. Brown, Aberration correction in the stem, Inst. Phys. Conf. Ser. (Proceedings EMAG97 meeting) 153 (1997) 35-40.

[17] R. Erni, Aberration-corrected imaging in transmission electron microscopy: An introduction (2nd edition), Imperial College Pressdoi:10.1142/p703.

[18] S. Pennycook, P. Nellist, Scanning transmission electron microscopy: Imaging and analysis, Springerdoi:10.1007/978-1-4419-7200-2. 
[19] R. Erni, M. Rossell, C. Kisielowski, U. Dahmen, Atomic-resolution imaging with a sub-50-pm electron probe, Phys. Rev. Lett. 102 (2009) 096101. doi:10.1103/PhysRevLett.102.096101.

[20] R. Erni, B. Freitag, P. Hartel, H. Müller, P. Tiemeijer, M. van der Stam, M. Stekelenburg, D. Hubert, P. Specht, V. Garibay-Febles, Atomic scale analysis of planar defects in polycrystalline diamond, Microsc. Microanal. 12 (2006) 492-497. doi :10.1017/S1431927606060594.

[21] C. Sun, T. Paulauskas, F. Sen, G. Lian, J. Wang, C. Buurman, M. Chan, R. Klie, M. Kim, Atomic and electronic structure of lomer dislocations at cdte bicrystal interface, Sci. Rep. 6 (2016) 27009. doi :10.1038/srep27009.

[22] C. Li, J. Poplawsky, Y. Wu, A. Lupini, A. Mouti, D. Leonard, N. Paudel, K. Jones, W. Yin, M. Al-Jassim, Y. Yan, S. Pennycook, From atomic structure to photovoltaic properties in cdte solar cells, Ultramicroscopy 134 (2013) 113-125. doi:10.1016/j.ultramic.2013.06.010.

[23] L. Li, Y. Chu, H. Li, L. Qi, Q. Fu, Periodically twinned 6h-sic nanowires with fluctuating stems, Ceram. Int. 40 (2014) 4455-4460. doi:10.1016/j.ceramint.2013.08.118.

[24] M. de la Mata, C. Magén, J. Gazquez, M. Utama, M. Heiss, S. Lopatin, F. Furtmayr, C. Fernández-Rojas, B. Peng, J. Morante, R. Rurali, M. Eickhoff, A. Fontcuberta i Morral, Q. Xiong, J. Arbiol, Polarity assignment in znte, gaas, zno, and gan-aln nanowires from direct dumbbell analysis, Nano Lett. 12 (2012) 2579-2586. doi:10.1021/nl300840q.

[25] M. de la Mata, C. Magén, P. Caroff, J. Arbiol, Atomic scale strain relaxation in axial semiconductor iii-v nanowire heterostructures, Nano Lett. 14 (2014) 6614-6620. doi:10.1021/nl503273j.

[26] T. Paulauskas, C. Buurma, E. Colegrove, B. Stafford, Z. Guo, M. Chan, C. Sun, M. Kim, S. Sivananthana, R. Klie, Atomic scale study of polar 
lomercottrell and hirth lock dislocation cores in cdte, Acta Crystallogr. Sect. A 70 (2014) 524-531. doi:10.1107/S2053273314019639.

[27] C. Li, Y. Wu, T. Pennycook, A. Lupini, D. Leonard, W. Yin, N. Paudel, M. Al-Jassim, Y. Yan, S. Pennycook, Carrier separation at dislocation pairs in cdte, Phys. Rev. Lett. 111 (2013) 096403. doi:10.1103/PhysRevLett.111.096403.

[28] A. Beyer, B. Haas, K. Gries, K. Werner, M. Luysberg, W. Stolz, K. Volz, Atomic structure of (110) anti-phase boundaries in gap on si(001), Appl. Phys. Lett. 103 (2013) 032107. doi:10.1063/1.4815985.

[29] M. Texier, B. Pichaud, M.-F. Beaufort, J.-F. Barbot, Structural disordering and extended defects produced by he-implantation in silicon carbide, J. Phys. D: Appl. Phys. 46 (2013) 485105. doi:10.1088/0022-3727/46/48/485105.

${ }_{540}$ [30] Y. Arroyo Rojas Dasilva, M. Rossell, D. Keller, P. Gröning, F. Isa, T. Kreiliger, H. von Känel, G. Isella, R. Erni, Analysis of edge threading dislocations $\vec{b}=1 / 2<110>$ in three dimensional ge crystals grown on (001)-si substrates, Appl. Phys. Lett. 107 (2015) 093501. doi:10.1063/1.4929422.

${ }_{545}$ [31] A. Kelly, K. Knowles, Crystallography and crystal defects (second edition), J. Wiley \& Sons, Ltd.doi:10.1002/9781119961468.ch10.

[32] J. Hornstra, Models of grain boundaries in the diamond lattice i. tilt about $<\overline{1} 10>$, Physica 25 (1959) 409-422. doi:10.1016/S0031-8914(59)94454-4.

[33] M. Vaudin, B. Cunningham, D. Ast, The structure and third order twin boundaries in silicon, Scripta Metall. 17 (1983) 191-198. doi : 10.1016/0036-9748(83)90097-2. 
[34] R. Banerjee, A. Bhattacharya, A. Genc, B. Arora, Structure of twins in gaas nanowires grown by the vapour-liquid-solid process, Philos. Mag. Lett. 86 (2006) 807-816. doi:10 .1080/09500830601055367.

[35] S.-G. Ihn, J.-I. Song, Y.-H. Kim, J. Lee, Gaas nanowires on si substrates grown by a solid source molecular beam epitaxy, Appl. Phys. Lett. 89 (2006) 053106. doi:10.1063/1.2245348.

[36] S.-G. Ihn, J.-I. Song, T.-W. Kim, D.-S. Leem, T. Lee, S.-G. Lee, E. Koh, K. Song, Morphology- and orientation-controlled gallium arsenide nanowires on silicon substrates, Nano Lett. 7 (2007) 39-44. doi:10.1021/n10618795.

[37] J. Johansson, B. Wacaser, K. Dick, W. Seifert, Growth related aspects of epitaxial nanowires, Nanotechnology 17 (2006) S355-S361. doi:10.1088/0957-4484/17/11/S21.

[38] J. Arbiol, A. Fontcuberta i Morral, S. Estradé, F. Peiró, B. Kalache, P. Roca i Cabarrocas, J. Morante, Influence of the (111) twinning on the formation of diamond cubic/diamond hexagonal heterostructures in cu-catalyzed si nanowires, J. Appl. Phys. 104 (2008) 064312. doi : 10.1063/1.2976338.

[39] L. Ramsdell, Studies on silicon carbide, Am. Mineral. 32 (1947) 64-82.

[40] A. Verma, P. Krishna, Polymorphism and polytypism in crystals, Wiley New York.

[41] C.-H. Wu, D. Aruguete, W. Reynolds Jr., M. Murayama, The role of twin boundary and surface energies in periodically twinned $<111>$ nanowires, Acta Mater. 75 (2014) 180-187. doi:10.1016/j.actamat.2014.04.048.

[42] A. van Helvoort, D. Dheera, H. Zhou, S. Grnsberg, G. Patriarche, B.-O. Fimland, H. Weman, Dark field transmission electron microscopy techniques for structural characterization of nanowire heterostructures, JPCS 241 (2010) 012084. doi:doi:10.1088/1742-6596/241/1/012084. 
[43] J. Johansson, J. Bolinsson, M. Ek, P. Caroff, K. Dick, Combinatorial approaches to understanding polytypism in iii-v nanowires, ACS Nano 6 (2012) 6142-6149. doi:10.1021/nn301477x.

[44] F. Bechstedt, P.Käckell, A. Zywietz, K. Karch, B. Adolph, K. Tenelsen, J. Furthmüller, Polytypism and properties of silicon carbide, Phys. Stat. Sol. (b) 202 (1997) 35-62. doi : 10.1002/1521-3951(199707) 202:1<35: :AID-PSSB35>3 . 0.C0;2-8.

[45] J. Bao, D. Bell, F. Capasso, J. Wagner, T. Martensson, J. Tragardh, L. Samuelson, Optical properties of rotationally twinned inp nanowire heterostructures, Nano Lett. 8 (2008) 836-841. doi:10.1021/n1072921e.

[46] D. Spirkoska, J. Arbiol, A. Gustafsson, S. Conesa-Boj, F. Glas, I. Zardo, M. Heigoldt, M. Gass, A. Bleloch, S. Estradé, M. Kaniber, J. Rossler, F. Peiró, J. Morante, G. Abstreiter, L. Samuelson, A. Fontcuberta i Morral, Structural and optical properties of high quality zinc-blende/wurtzite gaas nanowire heterostructures, Phys. Rev. B 80 (2009) 245325. doi:10.1103/PhysRevB.80.245325.

[47] T. Ito, Simple criterion for wurtzite-zinc-blende polytypism in semiconductors, Jpn. J. Appl. Phys 37 (1998) L1217-L1220. doi:10.1143/JJAP.37.L1217.

[48] T. Akiyama, K. Sano, K. Nakamura, T. Ito, An empirical potential approach to wurtzitezinc-blende polytypism in group iiiv semiconductor nanowires, Jpn. J. Appl. Phys 45 (2006) L275-L278. doi:10.1143/JJAP.45.L275.

[49] J. Hirth, J. Lothe, Theory of dislocations, Krieger.

[50] M. Kawabe, T. Ueda, Self-annihilation of antiphase boundary in gaas on si(100) grown by molecular beam epitaxy, Jpn. J. Appl. Phys 26 (1987) L944-L946. doi:10.1143/JJAP.26.L944. 
[51] O. Rubel, S. Baranovskii, Formation energies of antiphase boundaries in gaas and gap: an ab initio study, Int. J. Mol. Sci. 10 (2009) 5104-5114. doi:10.3390/ijms10125104.

[52] A. Beyer, I. Németh, S. Liebich, J. Ohlmann, W. Stolz, K. Volz, Influence of crystal polarity on crystal defects in gap grown on exact si(001), J. Appl. Phys. 109 (2011) 083529. doi:10.1063/1.3567910.

[53] M. Hytch, E. Snoeck, R. Kilaas, Quantitative measurement of displacement and strain fields from hrem micrographs, Ultramicroscopy 74 (1998) 131146. doi:10.1016/S0304-3991(98)00035-7.

[54] Y. Arroyo Rojas Dasilva, M. Rossell, F. Isa, R. Erni, G. Isella, H. von Känel, P. Gröning, Strain relaxation in epitaxial ge crystals grown on patterned $\mathrm{si}(001)$ substrates, Scripta Mater.doi:10.1016/j.scriptamat.2016.09.003.

[55] D. Hull, D. Bacon, Introduction to dislocations (fifth edition), Elsevier Ltd.

[56] M. Shetty, Dislocation and mechanical behaviour of materials, PHI Learning Private Limited, Dehli.

[57] A. Marzegalli, F. Isa, H. Groiss, E. Müller, C. Falub, A. Taboada, P. Niedermann, G. Isella, F. Schäffler, F. Montalenti, H. von Känel, L. Miglio, Unexpected dominance of vertical dislocations in high-misfit ge/si(001) films and their elimination by deep substrate patterning, Adv. Mater. 25 (2013) 4408-4412. doi:10.1002/adma. 201300550.

[58] S. Harada, J. Kikkawa, Y. Nakamura, G. Wang, M. Caymax, A. Sakai, 630 Vertical dislocations in ge films selectively grown in submicron si windows of patterned substrates, Thin Solid Films 520 (2012) 3245-3248. doi:10.1016/j.tsf.2011.10.092.

[59] C.-C. Chen, C. Zhu, E. White, C.-Y. Chiu, M. Scott, B. Regan, L. Marks, Y. Huang, J. Miao, Three-dimensional imaging of disloca- 
tions in a nanoparticle at atomic resolution, Nature 496 (2013) 74-77. doi:10.1038/nature12009.

[60] Y. Xin, S. Pennycook, N. Browning, P. Nellist, S. Sivananthan, F. Omnès, B. Beaumont, J. Faurie, P. Gibart, Direct observation of the core structures of threading dislocations in gan, Appl. Phys. Lett. 72 (1998) 2680-2682. doi : 10.1063/1.121097.

[61] H. Yang, J. Lozano, T. Pennycook, L. Jones, P. Hirsch, P. Nellist, Imaging screw dislocations at atomic resolution by aberration-corrected electron optical sectioning, Nat. Commun. 6 (2015) 7266. doi:10.1038/ncomms8266.

[62] S. Lopatin, S. Pennycook, J. Narayan, G. Duscher, Z-contrast imaging of dislocation cores at the gaas/si interface, Appl. Phys. Lett. 81 (2002) 2728. doi:10.1063/1.1511808.

[63] Y. Wang, P. Ruterana, S. Kret, J. Chen, S. E. Kazzi, L. Desplanque, X. Wallart, Mechanism of formation of the misfit dislocations at the cubic materials interfaces, Appl. Phys. Lett. 100 (2012) 262110. doi:10.1063/1.4731787.

[64] Y. Wang, P. Ruterana, The strain models of misfit dislocations at cubic semiconductors hetero-interfaces, Appl. Phys. Lett. 103 (2013) 102105. doi:10.1063/1.4820385.

[65] X. Wan, J. Liang, M. Liu, X. Jin, Dissociated screw dislocation which can relieve strain energy in the epitaxial layer of gesi on si(001), Phys. Rev. B 55 (1997) 9259. doi:10.1103/PhysRevB.55.9259.

[66] P. Lu, D. Smith, Dissociated $60^{\circ}$ dislocations in cdte studied by high-resolution electron microscopy, Philos. Mag. B 62 (1990) 435-450. doi:10.1080/13642819008215245.

[67] M. Ichimura, J. Narayan, Atomistic study of partial misfit dislocations in ge/si(001) heterostructures, Philos. Mag. A 73 (1996) 767-778. doi: $10.1080 / 01418619608242996$. 
[68] I. Kovács, L. Zsoldos, Dislocations and plastic deformation (first edition), Pergamon Press, Oxford.

[69] A. Cottrell, Theory of crystal dislocations, Gordon and Breach, New York.

[70] R. Erni, M. Rossell, M.-T. Nguyen, S. Blankenburg, D. Passerone, P. Hartel, N. Alem, K. Erickson, W. Gannett, A. Zettl, Stability and dynamics of small molecules trapped on graphene, Phys. Rev. B 82 (2010) 165443. doi:10.1103/PhysRevB.82.165443. 